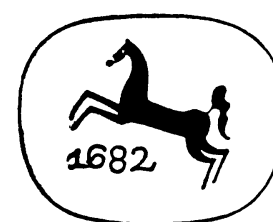

REIMSPRECHERKUNST IM SPÄTMITTELALTER 


\title{
Reimsprecherkunst im Spätmittelalter
}

\author{
EINe Untersuchung DER \\ TEICHNERREDEN
}

MCMLXX

J. B. Metzlersche Verlagsbuchhandlung

STUTTGART 
Additional material to this book can be downloaded from http://extras.springer.com

ISBN 978-3-476-99138-6

ISBN 978-3-476-99137-9 (eBook)

DOI $10.1007 / 978-3-476-99137-9$

(C) Springer-Verlag GmbH Deutschland 1970

Ursprünglich erschienen bei J. B. Metzlersche Verlagsbuchhandlung und Carl Ernst Poeschel Verlag GmbH in Stuttgart 1970 


\section{INHALT}

Vorwort .......................... IX

Abbildungen .... . . . . . . . . . . . . . . . XII

Wandlungen und Neuansätze in der Beurteilung spätmittelalterlicher

Literatur . . . . . . . . . . . . . . . . . . . . . . 1

I. Entstehung und Verwendung der Teichnerreden im Spiegel der Textüberlieferung

1. Die Teichner und der Teichner . . . . . . . . . . . . 14

Das Zeugnis der Schreiber und Sammler . . . . . . . . . . 14

Der Teichner-Schluß . . . . . . . . . . . . . . 18

Die Anziehungskraft der Teichnergattung . . . . . . . . 20

2. Doppelfassungen . . . . . . . . . . . . . . . . 22

Der ablösbare Beispiel-Eingang. Nr. 38 und Nr.684 . . . . . . . 23

Das zweiteilige Lehrstück. Nr.10 und Nr.689 . . . . . . . . . 27

Glaubenslehre in geschliffenem und in grobem Rahmen. Nr. 693 und

Nr.715 .. . . . . . . . . . . . . . . . . 30

Priorität der Fassungen und »Originalgedichte« . . . . . . . . 34

3. Mischredaktionen .. . . . . . . . . . . . . . . 36

Gedichtkomplexe aus Versatzstücken . . . . . . . . . 36

Seriendichtung und Vortragsauswahl . . . . . . . . . 45

4. Arrogate fremder Texte in den Teichnern . . . . . . . . . . . . 49

Aus dem Genre vom übelen wip. Überbietung anstelle von Originali-

tät . . . . . . . . . . . . . . . . . . . . . 49

Gesamtbeurteilung der Strickerpartien in den Teichnern . . . . 61

Zwei Stricker-Etüden des Teichners . . . . . . . . . . . . . 64

Konsequenzen für die Schulung des Teichners . . . . . . . . . . 72 
5. Reihendichtung und Reihenüberlieferung in den Sammelhandschriften 79

Die mehrstufige Lektion . . . . . . . . . . . . . . . . . . 79

Themenverschiebung innerhalb der Reihen . . . . . . . . . . . 82

Beteiligung der Sammler an der Reihenbildung . . . . . . . . . 83

Antithetisch und parataktisch gereihte Ständerügen . . . . . . . 87

Variabler Reihenbestand in verschiedenen Handschriften. Nr.63-72 89

Vortragseinheit und Überlieferungseinheit . . . . . . . . . . . 93

6. Sprecherspruch und Lesebuch . . . . . . . . . . . . . . 94

Das Auftrittsrepertoire .. . . . . . . . . . . . . 94

Improvisation und Gedächtnisvorrat. Der Weg des Vortragsrepertoires in die Sammlungen . . . . . . . . . . . . . . . . . . 98

Schreib- und Sammeleifer des Autors . . . . . . . . . . . 102

Interessenkreis der Sammler und Benutzer nach der Dokumentation der Handschriften . . . . . . . . . . . . . . . . . . 103

Das Lesebuch und seine Ordnung . . . . . . . . . . . . 106

II. Die Teichnerreden im Umkreis der zeitgenössischen Didaktik

1. Spätmittelalterliche Dichtung als Gebrauchskunst . . . . . . . . . 109

Tragweite und Grenzen des Begriffs . . . . . . . . . . . . . . 109

Zur Differenzierung der »bürgerlich-ständischen« Gesellschaft . . . 112

Die Rechtfertigung der Artistik . . . . . . . . . . . . . . . . 118

Der Teichner und die »einheitliche Kunstgesinnung« der Epoche . . 123

2. Reimsprecher und Spruchsinger . . . . . . . . . . . . 125

»Der künste koch«: Der Teichner und die sieben Künste . . . . . 125

»Des sanges zimmer«: Wort und Weise beim Teichner . . . . . . . 133

Ansätze zur sozialen Einordnung. Der Teichner und die Bruderschaften 137

Die »waren meister $\ll$ und » ein knoll der meister vein« . . . . . . . 141

3. Erzählkunst und Unterhaltungskünste im Blickwinkel des Sprechers . . 144

Die Lügenhaftigkeit der Aventiuren . . . . . . . . . . . . . . 144

Didaktische Kritik der Gattungen: >Seifrid Helbling`, Ottokar, Hugo von Trimberg, >Des Teufels Netz, der Marner, der Kanzler . . . . . 147

Literarische und ständische Konkurrenz . . . . . . . . . . . . 157

Die Gattungskritik in den Reimreden . . . . . . . . . . . . . . 159

Abstandnahme von der Epik als Gattungsregel. Konkurrenz der Un-

terhaltungskünste und Breite des Teichner-Publikums . . . . . . . 162

4. Die Eigenständigkeit der lehrhaften Reimreden . . . . . . . . . . 164

Abstandnahme von literarischer Konkurrenz. Anzeichen eines Werde-

gangs . . . . . . . . . . . . . . . . . . . 164

Die »Friedfertigkeit« des Teichners . . . . . . . . . . . . . . 167

Sprecheramt und geistliches Leben . . . . . . . . . . . . . . 172

Laienmissionierung in Österreich . . . . . . . . . . . . 175

Indizien des geistlichen Lehrauftrags . . . . . . . . . . . . . 177 
III. Grundlagen zu einer Poetik der lehrhaften Reimreden

1. Zur Aufgliederung der lehrhaften Reimreden . . . . . . . . . . 183

$»$ Liet« und $»$ Spruch $«$. . . . . . . . . . . . . . . . . . . . 184

Die Hohlform der Reimpaarkette . . . . . . . . . . . . . . . 186

Der Typenfächer der Spruchlyrik und die Reimreden . . . . . . . 188

$»$ Geistliche« und »weltliche« Rede . . . . . . . . . . . . . . . 194

2. Gemeinbesitz und Austausch literarischer Konventionsformen in der Lehrdichtung des späten Mittelalters . . . . . . . . . . Vergesellschaftung der Teichnerreden mit anderer Kleindidaktik in den Sammelhandschriften . . . . . . . . . . . . . Übergreifende literarische Konventionsformen: Tierfabel, Ständesatire, Aufreihekette usw. . . . . . . . . . . . . . . . . . 197

Gattungsstiftende und gattungsverbindende Tendenzen der Konventionsformen . . . . . . . . . . . . . . . . 200

Formenreichtum und Lehrhaftigkeit spätmittelalterlicher Dichtung . 202

3. Erscheinungsweise und Gesamtfunktion der literarischen Konventionsformen in den Teichnerreden . . . . . . . . . . . . . 204

3.1. Einführung und Anordnung der Rede

Die Beispielerzählung. Gleichnis und Parallelvorgang . . . . . . . 205

Vergleich und gestellter Fall . . . . . . . . . . . . . 208

Sentenzen als Auftakt und als Argument. Argumentation und Anschaulichkeit . . . . . . . . . . . . . . . 211

Hyperbolik und superlativischer Auftakt . . . . . . . . . . . . 213

Rangfragen. Sozialethische Abschattierung der Tugend- und Lasterbegriffe . . . . . . . . . . . . 215

3. 2. Dialog- und Erzählformen

Streitdialoge. Reduktion des Dialogs und Umschlag in Lehrrede . . . 219

Personalallegorie. Die Einebnung der allegorischen Zeichenhaftigkeit 222

Der Umgang mit Fabelmotiven. Erzählpointe und Auslegungspointe 226

Allegoresen . . . . . . . . . . . . . . . . . 232

Die Abkehr von allegorischer Darstellungskunst und die »Teichnerphase« des Suchenwirt .............. . 235

Die Reduktion epischer Formen. Der Lehrzweck der Erzählstücke . . 240

Die allgemeine Dienstfunktion poetischer Konventionsformen in der Tradition laienmissionierender Dichtung . . . . . . . . . 246

4. Die Lehrhaftigkeit der Reimreden . . . . . . . . . . . . . 248

4. 1. Die Ablösung der Lehrkunst von der Redekunst

Rhetorik und Homiletik . . . . . . . . . . . . . . . . 248

》Qui ergo docet, vitabit omnia quae non docent«: Sermo vulgaris und die »leichte predig" des Reimsprechers . . . . . . . . . . . . 252

Die Stände- und Lasterrüge. Praedicatio in verbo et in facto . . . . . 257 
Spiegelform und Wahrheitsgehalt der Lehre. Der Verzicht auf persönliche Anspielung und der Bekennermut des Reimsprechers . . .

4. 2. Die Entfaltung der Lehre in Predigt und Reimrede

Predigt, Traktat und Ansprache. Das Lesebuch zur Laienunterweisung 264

Predigtmäßige Eingangsformen in den Reimreden . . . . . . . . 268

Die Bedeutung des Exempels als homiletisches Hilfsmittel und als literarische Zweckform . . . . . . . . . . . . . . . . . 270

Der Aufbau der Argumentation: Die spezifischen modi dilatandi der Reimrede . . . . . . . . . . . . . . 274

Kompositionsformen der »weltlichen« Didaktik. Die consideratio contrariorum - Tugendlehre im Lasterspiegel . . . . . . . . . . . 281 Der Rangvergleich und die Kunst der correspondentia . . . . . . . 283 Abkehr von der Bispelrede und Aufnahme kausaler Argumentation. Konsequenzen aus der pragmatischen Verengung der exhortatio . . . 286 Die Erörterung praktischer Einzelfragen. Der Weg zur Narrendichtung und zu den turbae stultorum Geilers von Kaisersberg ... 290

Schluß

Anhang

Gesamtverzeichnis der Redaktions-Varianten in der handschriftlichen Uberlieferung . . . . . . . . . . . . . . 309

Bewahrung und Variation von Uberlieferungsgruppen in mehreren Handschriften

Suchenwirts Nachruf auf Heinrich den Teichner . . . . . . . . . . 321

Literaturverzeichnis

Texte ................... . . 324

Forschungsliteratur . . . . . . . . . . . . 329

Register

Teichner- und Stricker-Handschriften . . . . . . . . . . . . . 341

Texte der Teichner-Ausgabe . . . . . . . . . . . . . . . . . . . 343

Autoren .................. . 351

Schemata einzelner Überlieferungszusammenhänge . . . . . . . . . nach 359 
Die Verhältnisse, unter denen an deutschen Universitäten seit einem reichlichen Jahrzehnt Lehre und Forschung im Bereich unseres Faches betrieben werden konnten, sind mittlerweile so hinlänglich bekannt gemacht, daß man mir eine ausführliche Begründung für das jahrelang hinausgezögerte Erscheinen dieser Untersuchung ersparen wird. Sie wurde im Jahre 1960 als Habilitationsschrift abgeschlossen, schon damals in dem Bewußtsein, daß ein weiteres Vordringen in manchen Bereichen die eigene Befriedigung über das Erarbeitete vergrößert hätte. Vor allem nährten die Studien über den Zusammenhang von literarischen Konventionsformen und Gattungen und über die Auswirkungen des jeweiligen Verwendungszwecks auf Diktion und literarische Form ständig die Versuchung, die Arbeit nicht schon im Stande einer monographischen Behandlung der Teichnersammlung zu veröffentlichen. Inzwischen kann sie sich jedoch auf die Nachbarschaft von nahezu einem Dutzend achtenswerten, teils vorzüglichen Gattungsstudien zur Literatur des gleichen Zeitraums berufen, so daß die Festigung oder auch die Korrektur von seinerzeit mitunter gewagten Thesen nun leichter möglich war. Insbesondere sind die Stricker-Forschung und benachbarte Überlieferungsstudien, aber auch die weitere Erschließung der homiletischen Literatur dabei hilfreich gewesen. Wenn ich mich gleichwohl neben einigen Kürzungen und wenigen, notwendig erscheinenden Erweiterungen auf die Einarbeitung der inzwischen erschienenen Literatur beschränken konnte, so deshalb, weil sich in vielen Fällen die breitere Verwendbarkeit spezieller Ergebnisse und Ansätze so deutlich herausstellte, daß sich ihre zusätzliche Erhärtung im Bereich der Teichnerreden erübrigt. Das gilt insbesondere für die Beurteilung variabler Texteinheiten und -zyklen und für die Analyse predigtähnlicher Argumentationsformen. Hier hat die vorwiegend monographische Forschung der beiden letzten Jahrzehnte nunmehr eine hinreichend sichere Plattform geschaffen, um übergreifende Gegebenheiten, Zwecke und Wirkungsformen der spätmittelalterlichen Literatur in neuem und freierem Zugriff zu behandeln.

Nächst Werner Richter, der mir nach dem Abschluß meines Studiums mit einem 
Forschungsstipendium den notwendigen Arbeitsspielraum eröffnete, danke ich die Ermutigung, mich mit den Teichnerreden einzulassen, allererst Heinrich Niewöhner, der in vieljähriger gewissenhafter Arbeit die erste Teichner-Ausgabe zuwege gebracht hat. Nach dem Austausch von Korrekturen zur Einleitung der Ausgabe und von Lesarten seinerzeit nicht erreichbarer Handschriften stellte er mir bereits die Rohabzüge seiner Edition zur Verfügung, um die Last eigener Abschriften soweit wie möglich abzukürzen. Ich hätte gern diese Untersuchung, von der ich ihm nur erste Entwürfe zeigen konnte, noch in seine Hände gelegt. Ebenso gerne hätte ich mit Hanns Fischer die Ergebnisse unserer langjährig in benachbarten Gebieten betriebenen Arbeiten noch ausgetauscht. Von dem energischen Impuls seiner früheren Arbeiten mögen Abschnitte dieses Bandes selbst zeugen. Sein Buch über die Märendichtung kam mir im vorigen Herbst zu einem Zeitpunkt in die Hand, an dem der Textteil dieses Bandes schon abgeschlossen war. Eine Musterung der vielen Berührungspunkte, an denen die dort und hier vorgetragenen Ansichten einander ergänzen oder auch zu neuen Fragen Anlaß geben, erneuert immer wieder die Trauer um seinen frühen Tod.

Karl Stackmann, dessen unerbittliche Ermunterung den Fortschritt und den ersten Abschluß des Manuskripts am entschiedensten gefördert hat, wird auch die Erleichterung über das Erscheinen dieses Bandes mit mir teilen. Hilfsbereit hat Hugo Moser der Arbeit sein Urteil und seine Anregungen zugute kommen lassen. Aus Randnotizen Richard Alewyns erwuchsen neue Abschnitte oder bessere an Stelle der alten.

Ohne die Hilfe von Hilkert Weddige und Jürgen Schutte in Berlin wären zahlreiche Ermittlungen zur Erhärtung oder Vervollständigung der Quellenbelege auch bis heute nicht zustandekommen. Insbesondere waren die Reinzeichnung der Schemata, die Herstellung der Register und schließlich die mehrfache KorrekturLektüre nur dank der umsichtigen Mitarbeit von Barbara Fietkau, Ingrid Noblé, Ursula Rauh und Jürgen Schutte zu bewerkstelligen.

Die Mühe der Textermittlungen wurde mir schon während des Erscheinens der Teichner-Ausgabe und auch während der Revisionen bis in die jüngste Zeit erleichtert durch das Entgegenkommen aller befragten Bibliotheken, wobei ich der freundlichen und zuvorkommenden Unterstützung durch die Österreichische Nationalbibliothek in Wien besonders gedenken möchte. In Tübingen, München, Marburg, Heidelberg, Bonn und Berlin habe ich ebenfalls für bereitwillige bibliothekarische Hilfe zu danken.

Seit 1961 hielt die Deutsche Forschungsgemeinschaft großzügigerweise Zuschußmittel für den Druck bereit. Um so herzlicher kann ich dem Verlag meine Freude darüber bekennen, daß er sie schließlich nicht in Anspruch nahm, sondern Satz, Druck und Ausstattung auf eigene Hand aufs beste besorgte. 
Der Mann, der vor ziemlich genau sechshundert Jahren die erste auf uns gekommene Sammlung von Teichnergedichten niederschrieb, hatte einen doppelten Verdienst im Sinn. Am Schluß von fünfhundert wohlgefüllten Seiten bedachte er, $\mathrm{da} \beta$ er zwei Pfund und sechzig Pfennige vertan habe, um dieses Buch anzufertigen, und er war der Meinung, ein Käufer könne getrost drei Pfund Pfennige dafür geben,

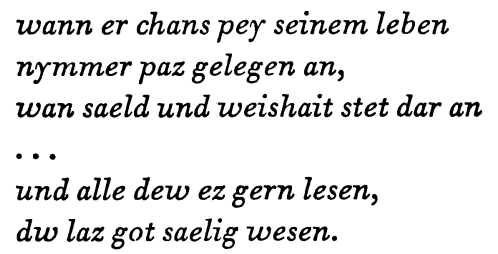

Ich wage nicht zu behaupten, daß von einem Buch über diese Gedichte im Jahre 1970 gleich heilsame Wirkungen ausgingen und da $\beta$ mit ihm ein vergleichbarer Gewinn zu erzielen sei. Allen denjenigen aber, die seine Niederschrift unter Aufwand von Zeit und Mühe mit beförderten, möge der wohlerwogene Geleitwunsch jenes Schreibers über die Jahrhunderte hinweg in vollem Maß zugute kommen, und allen Lesern der Gewinn, den sie erhoffen.

Berlin, im Juli 1969

E. L. 


\section{ABbILDUNGEN AUS EINER FRÜHEN UND EINER SPÄTEN TeichinersammLUNG}

Tafel II und III: Anfang und Schluß der dem Teichner zeitlich und regional nächststehenden Sammelhandschrift

Nationalbibliothek Wien, Cod. Vind. 2901 (= Teichner-Hs. A), fol. $4^{\mathrm{r}}$ und $249^{\mathrm{v}}$. Originalformat $30,1 \times 20,4 \mathrm{~cm}$ (unterer und rechter Rand von fol. $4^{\mathrm{r}}$ nachträglich schief beschnitten). Beide Seiten von demselben Schreiber, rote Verzierungen von einem zweiten Schreiber. Abgefaßt vor oder um 1370.

$\mathrm{Zu}$ fol. $4^{\mathrm{r}}$ :

Initiale rot, in der Füllung schwarzes Gitter mit roten Punkten; alle Buchstaben am Versanfang einzeln rot durchstrichen. Die Seite bietet 69 von 78 Versen des Gedichts Nr. 1, das auch die Sammlungen $E$ und $O$ eröffnet (Trinitätsformel!); ebenso stand es mit Sicherheit am Anfang von B, wo es jetzt durch Blattverlust fehlt.

$\mathrm{Zu}$ fol. $249^{\mathrm{v}}$ :

Alle Buchstaben am Versanfang von einem roten Strich durchzogen. Die Summenangabe am Schluß ganz in roter Schrift. Die Seite bietet die letzten 37 von 57 Versen des Gedichts Nr. 463, danach anstelle des durch Uberschrift angekündigten weiteren Gedichts die Abschlußverse, die die Handschrift zum Verkauf ausbieten (vgl. Gedichte I, S. XXXIII).

Tafel I und IV: Augsburger Sammelhandschrift aus dem späteren 15. Jahrhundert

Staatsbibliothek Preußischer Kulturbesitz Berlin, Ms. Germ. 564 (= Teichner-Hs. O), fol. $7^{\mathrm{v}}$ und $30^{\mathrm{v}}$. Originalformat $28 \times 19 \mathrm{~cm}$ (oberer Rand nachträglich beschnitten, Initiale »S« auf fol. $30^{v}$ deshalb unvollständig). Nach der Schlußnotiz des Schreibers Conrad Bollstatter (fol. 333 $) 1472$ abgeschlossen.

Zu fol. $7^{\mathbf{v}}$ :

Die beiden oberen Zeilen sind das Ende des Inhaltsverzeichnisses, das fol. $1^{\mathrm{r}}$ beginnt. Die angezeigte Rede „Von den vier Töchtern Gottes" gilt als Gedicht Heinrich Kaufringers.

$\mathrm{Zu}$ fol. $30^{\mathrm{v}}$ :

Die Schrift der Vorderseite $30^{\mathbf{r}}$ scheint durch. Die ersten drei Zeilen sind der Schluß des Gedichtes O 21. Nach der Zwischenüberschrift folgen 26 von 38 Versen des Gedichtes 022. Der hier in zwei Gedichten dargebotene Text ist in den Hss. A, B, E und P als ein Gedicht (Gedichte I, Nr.12), der zweite Teil in Hs. C mit einem anderen, langen Vorsatz (Gedichte III, Nr. 646) überliefert (vgl. das Schema III im Anhang). 


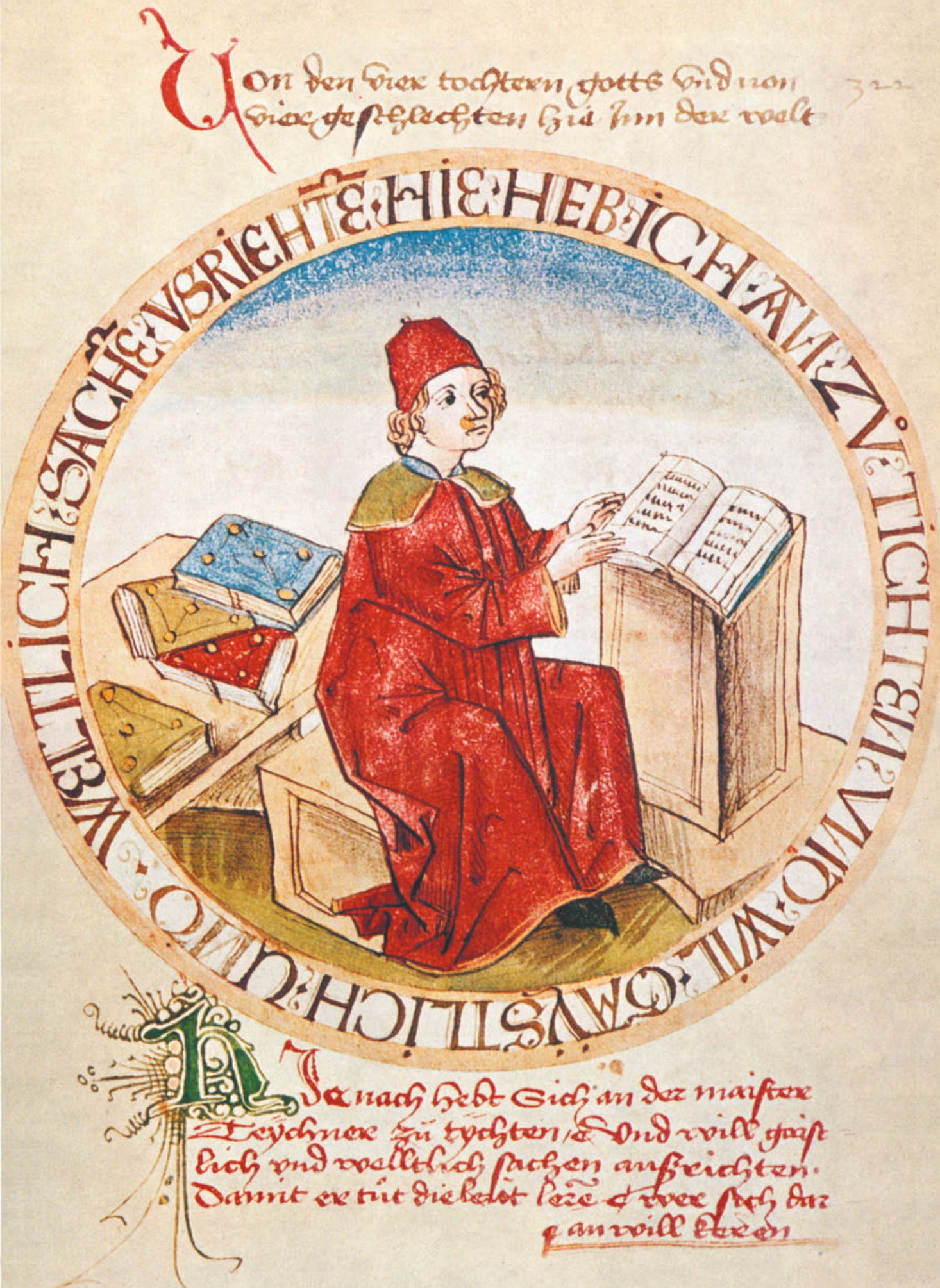




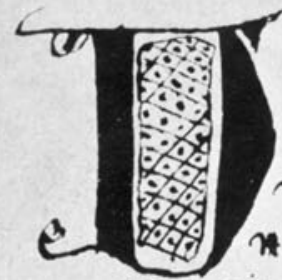



in grovitt an matiffaraft

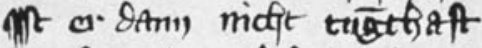

+\$o if matr ze pob $\theta_{1}$ in

क्रे vinb nit an qrior. mAn

काiter Omit $8 \mathrm{As}$, mitn in prere

Oowan or gevuaftigs if on ip 48

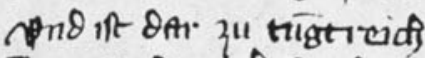

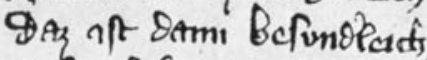
wol ze lobe von Sen zutum

Guvate vin nualfint lobr man dffam

Tos man anifit derriozon may

De- Reifig- gatit mi voffer. witg

it $i$ ibar Aflau Ointe Baburt ptat on 8 fin mar SA mit cirt wnt dea- Realug gatp geqreipt Ta-Batige gatit den vite nuapt

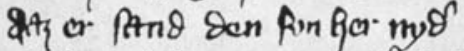



Sa unar mi allcpampt Bchba

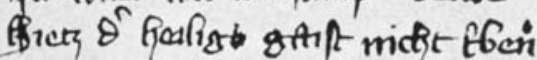

4. geat wn Audi m rifam rocpal

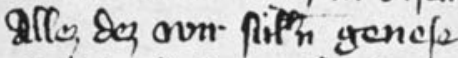

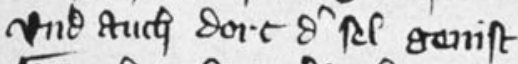

पot inf mat qu OAncion vuift

Das a mid befaraffen hiet $\$ 6$ pin grit mof migh buret

कo vater itf qu matien grit
No of Golig gatipt mitis miet

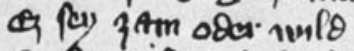



Nond it ungrefimpkes vol

Das or miaft gevele $\beta$ wol

$198 \mathrm{~cm}$ and Orind Sta nobe

Sem 8a- Ratig amp ift gebs

nuc pev pho Ainmit tirig.

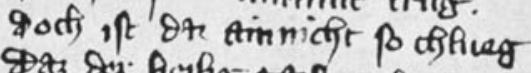

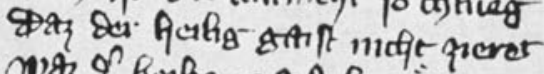

mets of Ruhg ganft Bevrict

43 py korts ade. $\operatorname{tn} \theta^{n}$ oind

4. of nof geftate uns rinct

中atoin in strge vno in mué



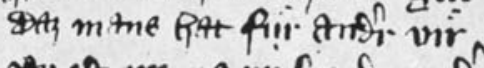

not if on parin fir. Sen tur on zer.

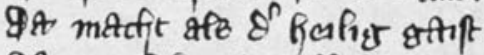

$\$ 8$ von bo mim afles migt

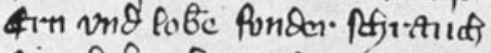

in cSef wof voref mit acpant?

PAntier aro $\mathrm{nme}$ cit $m$ aroz

- a dan Antar pin genozz

Scu on pirlo par mat.

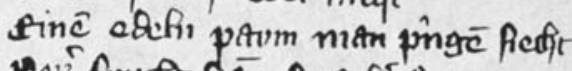

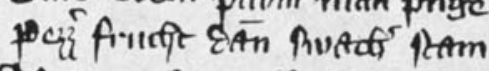

SA voll ift a afle a qam

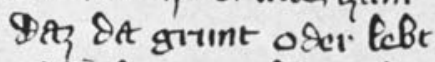

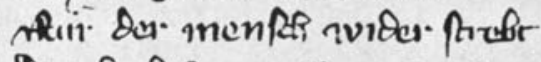

San Borf rent afle gepeaftr.



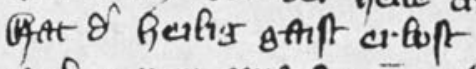

nire a pave une pemé noft

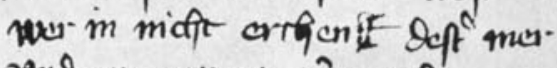

Ving $m$ crpour tiv ing a. of Gat vater vis fín viloren 
Sur wnt wor it wo soptefter

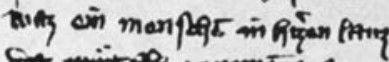
Sit mintitis ganoma the

to men m perfit moth Arue an Arues- cil angraion min

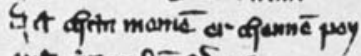

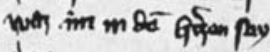
Womms as enter pafonyt ticit Q0 it $m$ Afsur. worp ju mir Qes Sty fine furmamiont wiक on afla-veptet prowt Do nues an ungavive prevn Atp mand dan ungariun Wivin of prount in putce grite



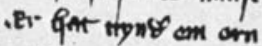

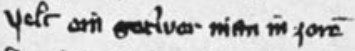

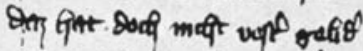
A grover in an Gitir nof in $\theta$ qutef nit tous mit irrit Awer on imgarivon mit of ip pitar $m$ iñ pain की वीAn munet in pant gle AniB ponome pain grontuis

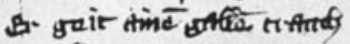

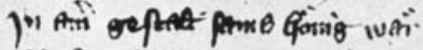

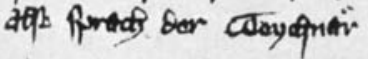

\section{- Toum boum port nopar}

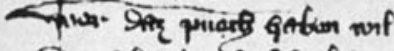

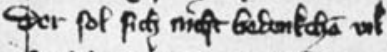

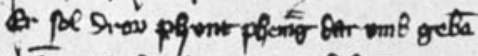

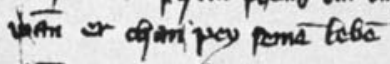

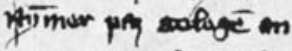

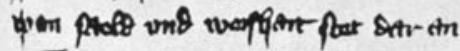
Gor inft in oes Peba pin

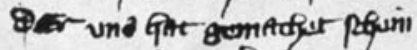
uno atio in of in espon
Qwe on portig puep

19 erin an far tuor diane

19i form An- 06 qwaty $\mu$ Rint

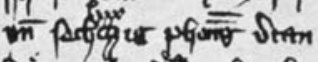



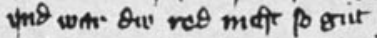

- vono mafpar in mone miir

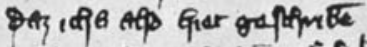

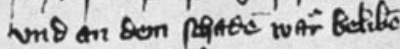

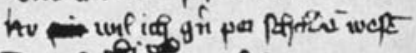

Dis uf की

Drant ant ain sno

Sole und pain gerato put

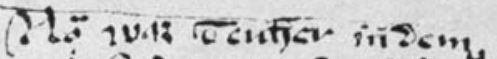

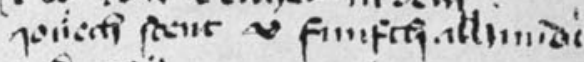
जाis vinj 


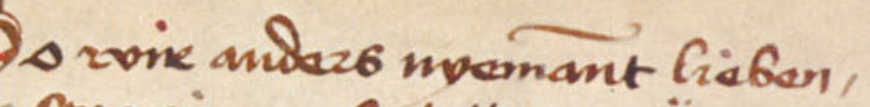

Dopy won ances gatt vmuine

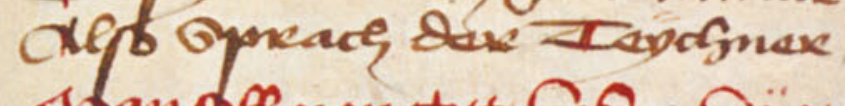

inban ba non dott raber boiven figen

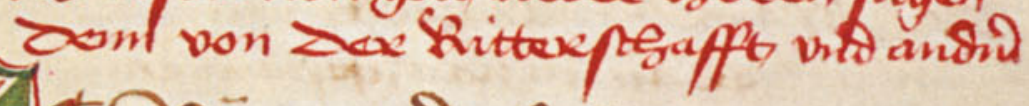

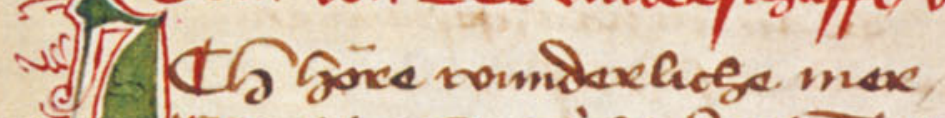

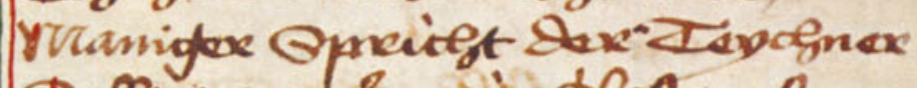
\$ort nave Imm inistloptex forer

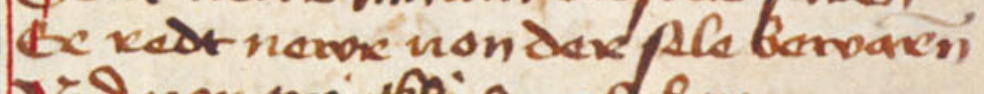
Vind non uvightigem debul

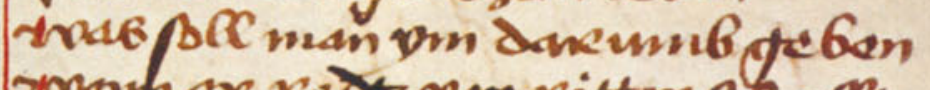

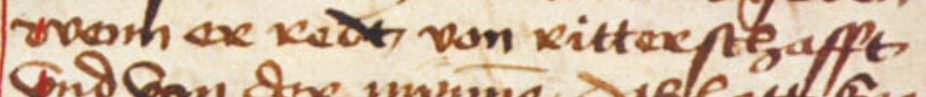

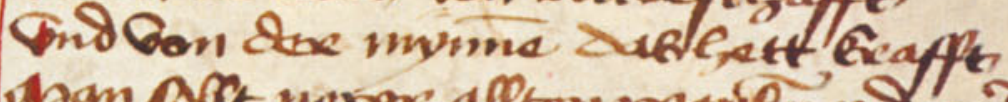

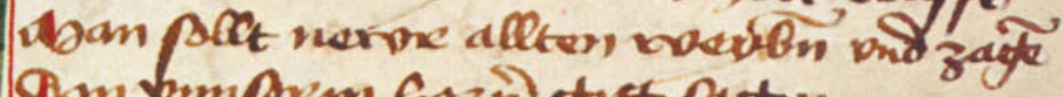
Dam bimparm sozin gatat paten!

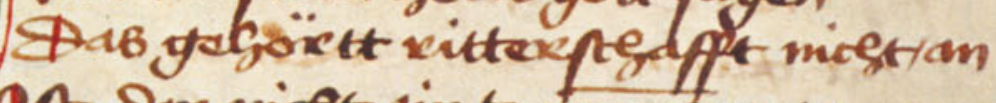
op Sex mist ain tummex mum.

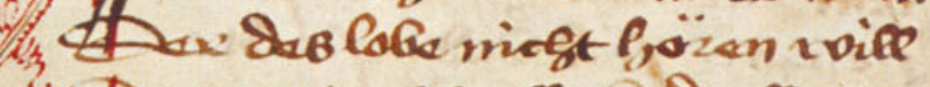

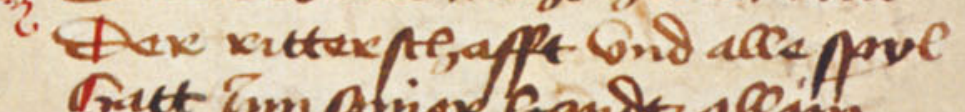
Gutt im plined hamst aleim




tind voas tugtent amimigh

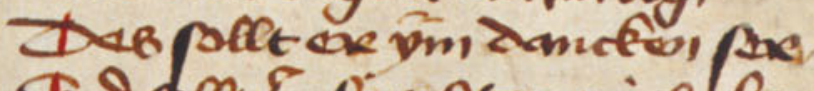

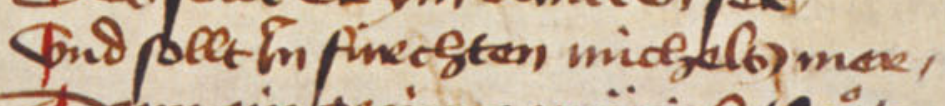

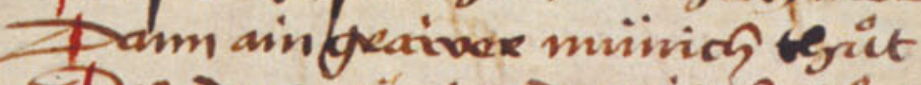
Ales dax minmice, dox ip belpitt

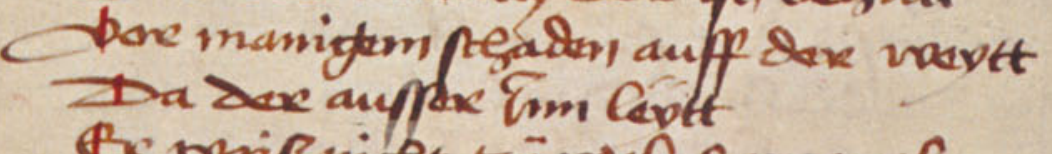
Ac raif nicht sü ndaceor mal,

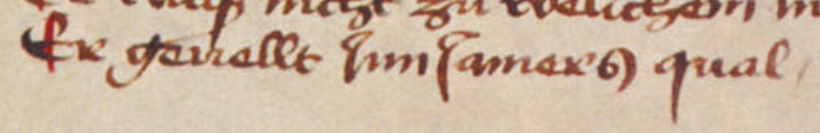

